\title{
The Need for and Development of Behaviourally Realistic Agents
}

\author{
Wander Jager ${ }^{1}$ and Marco Janssen ${ }^{2}$ \\ ${ }^{1}$ Faculty of Management and Organisation, Dept, of Marketing, University of Groningen. PO \\ Box 800, 9700 AV, Groningen, The Netherlands. w. jager@bdk.rug.n1 \\ ${ }^{2}$ Center for the Study of Institutions, Population, and Environmental Change, Indiana \\ University. 408 North Indiana Avenue \\ Bloomington, IN 47408-3799 USA. maajanss@indiana. edu
}

\begin{abstract}
In this paper we argue that simulating complex systems involving human behaviour requires agent rules based on a theoretically rooted structure that captures basic behavioural processes. Essential components of such a structure involve needs, decision-making processes and learning. Such a structure should be based on state-of-the-art behavioural theories and validated on the micro-level using experimental or field data of individual behaviour. We provide some experiences we had working with such a structure, which involve the possibility to relate the results of simulations on different topics, the ease of building in extra factors for specific research questions and the possibility to use empirical data in calibrating the model. A disadvantage we experienced is the lack of suiting empirical data, which necessitates in our view the combined use of empirical and simulation research.
\end{abstract}

\section{Introduction}

Reynolds [1] developed an approach to simulate flocks as a distributed behavioural model. His work on the flocking boids has become a key example how simple local rules lead to complex macro behaviour. Reynolds used three rules for each agent: avoid collisions with nearby flockmates, attempt to match velocity with nearby flockmates and attempt to stay close to nearby flockmates. Due to measurement errors by the flockmates, an impressive flocking like behaviour comes out of this model. Reynolds mentions that "success and validity of these simulations is difficult to measure objectively. They do seem to agree well with certain criteria and some statistical properties of natural flocks and schools which have been reported by the zoological and behavioural sciences. Perhaps more significantly, many people who view these animated flocks immediately recognize them as a representation of a natural flock, and find them similarly delightful to watch" [1, p.26].

One might derive the impression that we have a better understanding of flocking behaviour. However, research on schooling of fish illustrate that we lack a good understanding of the micro-behaviour of fish in relation to schooling. Indeed, information about the behaviour of nearby neighbours is found to be a crucial factor 
in empirical studies, but which behavioural rules are in use is a puzzle and so far computational models fail to reproduce observed behaviour in detail [2].

Reynolds showed that simple local rules could be used to simulate interesting macro-behaviour. As a computer scientist, he was not studying how flocking behaviour was happening in the real world. If that was the case a more rigorous analysis of the micro-behavioural rules tested in controlled experiments would have been necessary. In fact, this is what is happening in biology. In [2] an excellent overview is given on the study of self-organization in biological systems. It shows that the puzzles in their field are recently successfully being approached by combining field work, controlled laboratory experiments and models. They stress that the models "should be developed solely based on observations and experimental data concerning subunits of the system and their interactions" [2, p.70].

If we look at the use of multi-agent models for the study of social phenomena, we have to conclude that the fruitful combination of fieldwork, laboratory data and modelling is lacking. Key publications in social simulation like segregation by Schelling [3] and the evolution of cooperation by Axelrod [4] could explain macro-phenomena, by assuming simple logical rules for the behaviour of the agents. However, like the flocking boids the behavioural rules are not validated by empirical research or based on decision-making theories. We do not want to reduce the importance of the contributions of Schelling and Axelrod, they are evidently milestones. No, we want to argue that the use of simulation models should more often be based on empirically tested theoretical models of human decision-making combined with rigorous empirical research in the field and in the laboratory. This is happening in some areas, although they are often not included in the work of multiagent simulation. A nice example of such a combination of empirical and simulation research is done by Erev \& Roth [5]. They studied learning in experiments in games with a unique mixed strategy. Using a large data set of experimental data, they calibrated different versions of reinforcement learning and tested its predicted value. They showed that reinforcement learning model robustly outperforms the equilibrium predictions. Duffy [6] performed experiments on speculative behaviour with simulated agents, which were modelled on the basis of prior evidence from human subject experiments. Hommes [7] uses experimental data on financial markets in his simulation models of market behaviour. Gigerenzer et al. [8] study which theoretically-sound heuristics explain the observations in laboratory experiments.

Rational choice theory is one of the dominant theories in social science. Although it might often be nicknamed as Homo economicus, the rational agent is also used in other social sciences than economics as the theoretical construct of human decision-making. There is an increasing amount of anomalies of the rational choice theory found in experimental work. For example, subjects cooperate, when theory predicts defection, and framing of the problem leads to different results of the decision [9]. Nobel Laureate in Economics Reinhard Selten states "Modern mainstream economic theory is largely based on an unrealistic picture of human decision-making. Economic agents are portrayed as fully rational Bayesian maximisers of subjective utility. This view of economics is not based on empirical evidence, but rather on the simultaneous axiomisation of utility and subjective probability." [10, page 13]. 
Many researchers thus felt uncomfortable with the rational-actor assumption, and started to experiment with 'bounded rational' formalisations of human choice behaviour. These researchers were not satisfied with using a model that yielded the same outcomes but on the basis of different processes (heterologous or analogous metaphor, see e.g. [11]). Rather they were interested in trying to capture the same laws that apply to real human behaviour into agent rules (unificational metaphor). However, searching for generic laws of human behaviour quickly causes one to get lost in the abundance of psychological theories on motivation, emotions, norms, social comparison, imitation, habit formation, attitudes, and many more. So instead of making a Don Quixoteian endeavour in capturing all those theories in a single agent, they adhered to the adagio of 'keep it simple, stupid' in order to keep the model simple and the simulation results transparent for interpretation. Hence many simple formalisations of bounded rational agents were and are being developed and tested in different virtual environments. Most of these models demonstrated clearly that different assumptions of agent rules on the micro level had serious consequences for the outcomes at the macro level. A very neat series of experiments was performed already in 1994 by Bousquet et al [12], who gradually increased behavioural realism in the decision-making process of a society of fishermen. Currently François Bousquet and his colleagues use role games to extract rules-in-use by the community of interest [13].

Yet, looking at most formalisations of 'bounded rationality' in agent rules they leave the impression of being developed rather 'ad hoc' from the perspective of programming rules rather than reflecting a formalisation on the basis of theoretical considerations. Bounded rationality is not an excuse for using sloppy decision rules. For example, it is very interesting to study the effects of introducing an 'imitation' strategy in agents within a system. However, not considering the issues of under what conditions the agents are likely to imitate, and which other agents they are most likely to start imitating, may yield results that do not originate from the 'psychological laws' on imitative behaviour.

A number of psychologists have started to formalise existing theories in agent rules as to explore the derived behavioural assumptions in a more dynamical context. A very nice example is the formalisation of the Elaboration Likelihood Model by Mosler, Schwarz, Ammann and Gutscher [14]. Here the simulation contributed to the dynamic shifting of attitudes as depending on processing intensity. Also in the fields of social cognition, emotion, social behaviour and normative behaviour more and more simulation models are being used as a complementary tool to study how these empirical validated 'psychological laws' perform in a dynamical context. Returning to human behaviour in complex systems, we may conclude that many behavioural theories may be of importance at different moments in time. People perform habits, they will change their attitudes, develop habits and the like, and all these processes have been described in psychological theories. In fact, we should not only look at theories from psychology, but also integrate theories from other disciplines. Wilk [15] discusses how different approaches to study consumer behaviour, like individual choice theories, social theories and cultural theories, lead to different insights and different policy recommendations. Hence, many theories should be formalised in agent rules as to capture the spectrum of human behaviour in agent rules. However, the many theories, - formalised in simulation models or not (yet) would yield a far too complex agent as to keep the model programmable and the 
results transparent for interpretations. Moreover, it would be a very tricky task linking the various theoretical formalisations together in a theoretically and conceptually sound manner. This is especially difficult because so many psychological theories show overlap or describe partly the same processes at a different aggregation level. To do so, we state that a simplified meta-theory is required that organises the various theories in a conceptual framework. Vallacher \& Nowak [16] have already identified the need for such a meta-theory. Such a meta-theory would be helpful in developing agent rules that are aimed to capture a broad spectrum of human behaviour in a very simplistic manner as to represent the full spectrum of human behaviour in simulating complex systems. Three main issues that deserve critical attention in the development of such a meta-theory are agent rules are needs, the decision-making process and learning. We will discuss these issues separately in the next sections.

\section{Agent Needs}

In our daily lives we often have to compromise between conflicting interests. For example, many people find it difficult to find a good balance between working and spending time with family and friends. Every action we perform costs time, which decreases the opportunity to perform other actions. In our view this compromising reflects the multidimensionality of human needs: different actions may satisfy different needs, and in the end we try (we do not always succeed) to make decision over different activities such as to satisfy our various needs. This matches with theoretical conceptions of needs in psychology and economy (e.g., [17], [18]). For example, Maslow [17] discerns physiological and safety needs, needs to belong and be loved, and esteem, cognitive, aesthetic and self-actualisation needs. Yet many artificial agents are (implicitly) equipped with a single need, because they 'live' in a simple one-dimensional world, where only one 'good' can be consumed. This good is formalised in terms of abstract points, and an agent is implicitly assumed to have an everlasting motivation to consume more of these points, irrespectively of the number it has consumed before. Consequently, the agents are equipped with a single unsatisfiable need for points. The first point consumed is considered to be equally satisfying as the consumption of point number 100. Thus, a next unit of goods will contribute the same to the agents need satisfaction, independent of the number of goods consumed previously. This formalisation does not allow for the modelling of conflicting interests between different needs.

Forty years ago, Sauermann and Selten [19] presented a framework to tackle the problem of making decisions when you have different needs, in their approach different aspirations, without transforming the needs into one utility function. In [20] a more formal approach was presented, but so far this approach never been operationalised. Hence, an interesting task for the MAS community.

The modelling of different needs is in our view very important as to avoid this 'single need maximisation' of agents. This does not require that agents will be equipped with e.g., the seven needs as distinguished by Maslow [17]. Formalising two needs already allows for conducting experiments where agents have to compromise. For example, formalising a need for belongingness would stimulate agents to conform 
to the actions of the group, whereas a need for identity would stimulate deviant actions. Hence the two needs may require different actions for their satisfaction, implicating that the agent has to compromise.

\section{Agent Decision-Making}

Human structures, as well as human psychological mechanisms, at some fundamental level of description, can be analysed in terms of the problems they solve [21, p 321]. For example, social adaptive problems were so crucial for human survival and reproduction, that many of the most important features of our evolved psychological mechanism will necessarily be social in nature [21, p 323]. As a consequence, we developed socially and individually oriented psychological mechanisms that help us in making decisions, such as imitation, forming habits and following norms.

The various psychological mechanisms we have developed to solve problems are called 'heuristics'. These heuristics simplify complex decision problems, and save on cognitive effort involved in a decision. The work of Simon [22] on bounded rationality offers a perspective on why habits and complying with a norm may be a rational thing to do. The essential argument is that humans optimise the full process of decision-making (procedural rationality), not only the outcomes (substantive rationality, [22]). This holds that people may decide that a certain choice problem is not worth investing a lot of cognitive effort, whereas another choice problem requires more cognitive attention. Hence it is possible to describe the conditions that favour the use of certain types of heuristics. This would be helpful in formulating bounded rationality in artificial agents as a set of conditions that determine the use of certain strategies. For example, in formalising an imitation rule in an agent, one would also be able to formalise under what conditions this strategy will be employed.

In the last years, we have made considerable effort in trying to develop a framework to organise various heuristics [23], [24]. Here, we make a distinction regarding the cognitive effort that is being invested in the decision-making process, and the degree to which social information is being used. Generally, the less important a decision problem is (low contribution to multiple need satisfaction), the less cognitive energy (time) one is willing to invest in the decision, and hence, the simpler the heuristic that will be employed. Next to that, the degree of social information being used in heuristics differs significantly. Often people rely on their own previous experiences in a heuristic. For example, they may simply repeat previous behaviour. On the other hand, people may effectively use their cognitive capacity in considering the behaviour of others as to quickly find out attractive opportunities. Hence it appears that the heuristics that people employ can be organised on two dimensions: (1) the amount of cognitive effort that is involved, and (2), the individual versus social focus of information gathering [23].

In organising the various heuristics that people employ, we find it instructive to use the two dimensions of cognitive effort and proportion of social processing as depicted in Figure 1 (see e.g., [24]). Here, C stands for the cognitive effort invested in a decision heuristics, and $\beta$ for the contribution of social information. In the figure we positioned several heuristics. 


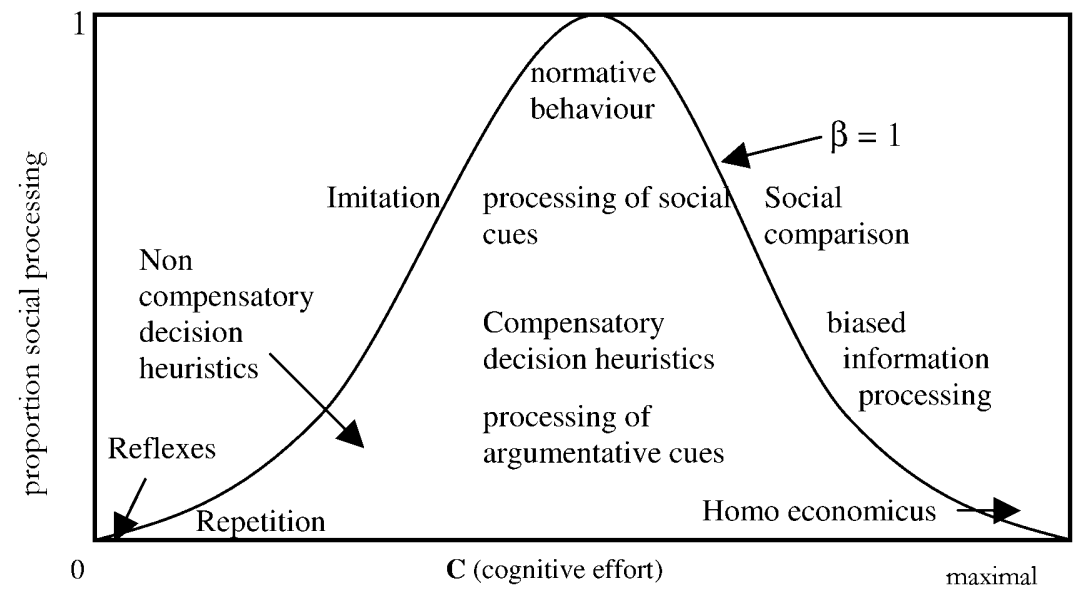

Fig. 1. Different heuristics organised along the dimensions of cognitive effort and use of social information

Figure 1 shows that heuristics that hardly require any cognitive effort (reflexes), or require very much cognitive effort (the prototypical homo economicus resembling the rational actor) do not use social information. Strategies that require an intermediate cognitive effort may use both social and non-social information. Here, uncertainty is a key factor that determines the degree to which social information is being used in the decision-making process.

The selection of a heuristic may not necessarily be conscious [25], but be an automaticity that has been learned (education, experience and looking at other people) and inherited (personality). Hence in the formalisation of agent rules it is possible to formalise under what condition which heuristic will be used.

Note that decision-making heuristics determine how agents make a choice, not what they choose. One of the main puzzles in social science is non-selfish behaviour observed in laboratory experiments and field work. This can be explained by need satisfaction where social value orientation, such as cooperative and competitive attitudes can be an important factor.

\section{Agent Learning}

People interacting within a system will learn about the system and the behaviour of other people, and may use this learned information in their decision-making. For example, after experiencing a number of droughts, people may have learned that occasional droughts belong to the behaviour of the natural system. Or a fisherman may learn from observation that cheating on the harvest quota may yield a substantial extra income. On the basis of this learning from system behaviour and other people's behaviour, people may decide on their own actions. For example, they may store food for droughts, exceed their harvest quota, or, on the contrary, decide to address the other cheater on his immoral behaviour. The latter example shows that learning may be a precondition for the emergence of norms. 
Having learned what a proper action is in a given situation diminishes the necessity of investing as much cognitive effort the next time the same situation is encountered.

Learning may occur in situations where more or less cognitive effort is being invested. For example, by experiencing certain outcomes after performing particular behaviour a behaviouristic learning may occur, where the strength between the two events gradually increases. On the contrary, a person carefully scrutinising a problem may suddenly discover a relationship, and by means of this cognitive learning immediately experience a strong connection between two events.

Many agents that have been formalised up till now have no or only a very limited learning capability, including the consumat approach we developed. However, also agents have been developed that primarily focus on developing a cognitive structure that allows for a theoretical valid way of modelling processes of learning (e.g., [26], [27], [28], [29]). What is necessary for this learning is a memory in which the agent's perception of the system is being represented. As the understanding of a system can be represented in terms of the combined occurrence of certain events, such a memory may take the form of a neural network, which is in fact a stylised model of the real human brain. The principle that guides the learning process can be described using Hebb's learning rule [30]. This learning rule describes connection growth and - strengthening as a consequence of simultaneous activity of two neurons. Replacing the concept of neurons by events implies that the closer the occurrence of two events, e.g. behaviour and consequences, the stronger these two events will be connected. On the other hand, when two events cease to occur together, the connection between these two events also decreases. Hence, formalising the memory of agents in such a way allows for continuous learning in a changing system. Moreover, modelling the memory using a neural network approach allows for combining a continuous learning process with relative straightforward heuristics as derived from theory on decision-making.

\section{The Need for Integration}

In the previous sections we discussed needs, decision-making and learning as critical components of a meta-model of behaviour. These components are very strongly connected. For example, a person may be hungry during a famine (low need satisfaction), and therefore invest a lot of cognitive effort in finding new ways of storing food (decision process), and discovering a new way of conserving vegetables over a long period of time (learning). Learning this new way of storing food also diminishes the urgency of the problem and of investing a lot of cognitive effort in satisfying the need for food. Other persons may observe this new food storage technique, and decide to adopt it. These people learn to satisfy their need for food by using social heuristics. Hence it is clear that needs, heuristics and learning processes should be considered in combination in understanding how people adopt themselves to the system they take part of.

Also when formalising artificial agents that take part of a larger system, it is necessary to address the questions of needs, heuristics and learning, and especially to explicate how these issues are related in the model. Here the model builder is faced with the challenge to pick those theories from the abundance of information that are hypothesised to play a crucial role in the system behaviour under survey, and translate them into simple agent rules that still have a clear relation with the original theory. Hence, 
models that are focussing on different systems may employ agent rules that differ with regard to the formalisation of needs, heuristics and learning processes, and yet are based on the same basic structure. In Figure 2 we propose such a basic structure that can be used to formalise agent rules.

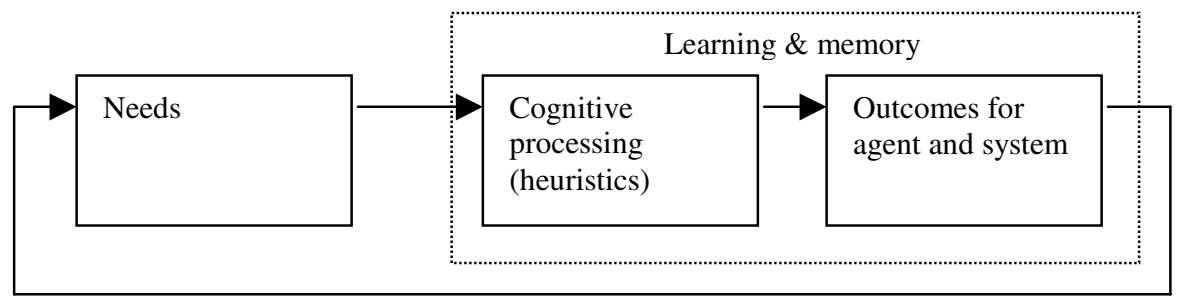

Fig. 2. A basic structure of artificial agents in complex systems

Essential in this basic structure is the feedback loop. This loop involves that the outcomes of behaviour, both on an individual level as on a system level, feedback to the individual needs. For example, the decision to over-harvest may cause a surplus of food now (individual) and a depletion of the food resource (system), which may satisfy the need for food now, but not in the long run.

\section{An Example: The Consumat Approach}

In recent years we developed the consumat approach as a tool aimed at the integration of various behavioural theories in a relative simple agent architecture [23], [31]. In [23] a review of relevant theories is provided, as well as a formalisation of various theories. The consumat approach emphasised especially the role of different needs and cognitive processes (heuristics), whereas learning processes have hardly been the subject of elaborate conceptualisation. The consumat approach has now been applied to common-pool problems [32], [33] and market dynamics [34], [35]. Like the boid flocking, we mainly showed in our previous publications that differences in needs and cognitive processes matters. But unlike the boid flocking our starting point was to define rules on agents based on theories from psychology. Although a number of theoretical problems are still waiting to be challenged, we now shifted our focus combining our theoretical agents with laboratory experiments and field work. In [36] we report on using the consumat approach to explain a set of laboratory experiments on common-pool resources.

All these simulation experiments are based on the same agent architecture. That is, first we formalise a number of needs. Here, we use the empirically grounded taxonomy of human needs as developed by [18]. This taxonomy comprises nine needs: subsistence, protection, affection, understanding, participation, leisure, creation, identity and freedom. Because formalising nine needs in an agent yield a very complex agent, we condense two or three needs out of this set to implement in the agent. Often we use a personal need, a social need and a need for identity. The personal need relates to how 
well a specific behaviour (product) satisfies the personal needs for the agent, and hence relates to nutritional value of food or the match of product characteristics with personal preferences. Next, the social need expresses the agent's preference to be in the neighbourhood of other agents (physical distribution) and/or to consume the same products/opportunities as other (similar) agents. Finally, the status need expresses the agent's need to possess more food, goods or capital than other agents (in the neighbourhood). Formalising different needs is essential to model conflicting interests in human behaviour. Instead of (implicit) single need agents, which ultimately try to maximise the satisfaction for this single need, the consumats may be confronted with trade-offs between needs. For example, the personal need may be a motivator to go to a location with a lot of food and a few other agents, whereas the social need may motivate the agent to go to a location where many other agents are, and where food may be less abundant. An aggregated level of need satisfaction is calculated by a weighted summing of the various needs. To express heterogeneity in agent's need valuation it is possible to attach different weights to the different needs in the weighted sum.

Another critical variable in the consumat is uncertainty of the agent. Uncertainty is a key factor that promotes the use of social processing in human beings (e.g., 37). In the consumat approach we formalise uncertainty often as a function that comprises both the expected outcomes and the actual outcomes. The larger the difference (over time) between these values, the larger the uncertainty gets. It is also possible to formalise uncertainty directly as a function of the perceived resources. In this way oscillations in e.g. food availability may cause uncertainty.

The level of need satisfaction and uncertainty determine the type of decision strategy an agent engages in. Critical factors here are the aspiration level and the uncertainty tolerance of the agent. Aspiration level indicates with what level of need satisfaction the agent is satisfied. Agents with a low aspiration level will be easy to satisfy, and hence do not engage quickly in intensive processing. On the contrary, agents with a high aspiration level are hard to satisfy and invest a lot of cognitive effort in their decisionmaking process. Aspiration level can conceptually be linked to personality traits that have been described in the Abridged Big Five Dimensions Circumplex of personality traits [38]. Uncertainty tolerance also fits very well on this taxonomy of personality traits. People having a low uncertainty tolerance are more likely to look at other people's behaviour, whereas people having a high uncertainty tolerance are more self-confident, and less sensitive to other people's behaviour.

Depending on the multiple need satisfaction of the agent (state), the agent's aspiration level (trait), the uncertainty of the agent (state) and the uncertainty tolerance of the agent (trait) it may engage in six decision rules that resemble cognitive processes (heuristics). Consumats having a very low level of need satisfaction are assumed to deliberate, that is: to determine the consequences of all possible decisions given a fixed time-horizon in order to maximise their level of need satisfaction. Consumats having a medium low level of need satisfaction and a low degree of uncertainty are assumed to engage in satisficing. This implies a strategy where the agent determines the consequences of decisions one by one, and selects the first decision that satisfies its needs. Consumats having a medium low level of need satisfaction and a high degree of uncertainty are assumed to engage in social 
comparison. This implies comparison of its own previous behaviour with the previous behaviour of consumats having roughly similar abilities, and selecting that behaviour which yields a maximal level of need satisfaction. Consumats having a medium high level of need satisfaction and a low degree of uncertainty are assumed to engage in improving. This implies determining the consequences of decisions one by one, and selecting the first decision that improves its need satisfaction. When consumats have a medium high level of need satisfaction, but also a high level of uncertainty, they will imitate the behaviour of other similar consumats. Finally, consumats having a very high level of need satisfaction simply repeat their previous behaviour. In the following Figure 3 it can be seen that these six rules resemble the organisation of heuristics as depicted in Figure 1.

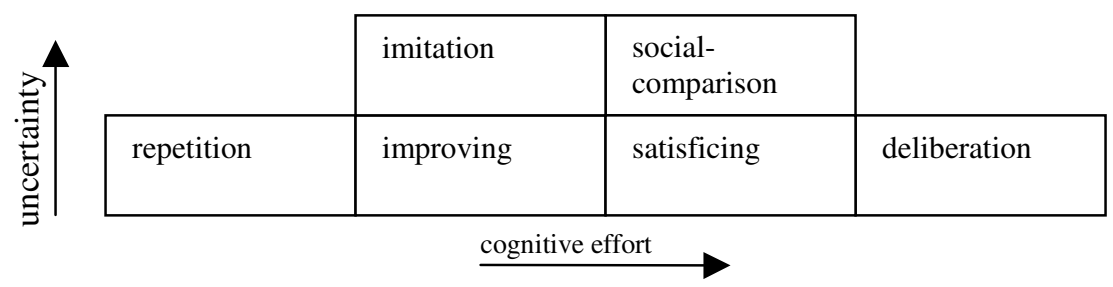

Fig. 3. The six decision rules as used in the consumat approach

When consumats invest more cognitive effort in their decision-making (deliberation, satisficing and social comparison) they will update the information in their mental map, which serves as a memory to store information on abilities, opportunities, and characteristics of other agents. This can also be understood as a more cognitive learning style. When consumats invest less cognitive effort in their decision-making (repetition, improving and imitating) they do not update their memory before making a decision. However, in as much the resulting outcomes will change the strength between events in the memory a more behaviouristic learning process will take place.

Modelling the six rules as requiring different quantities of time allows for the formalisation of cognitive effort. Here repetition costs hardly any time (close to $0 \%$ of the maximum), improving and imitation somewhat more (say 33\%), social comparison and satisficing even more (say 66\%) and deliberation the most (100\%). The important consequence of such a formalisation is that the use of simpler rules may yield better outcomes due to the saving of time. For example, imitating the foraging behaviour of others may cause one to obtain a satisfactory quantity of food. Whereas a deliberating agent may find richer opportunities, the associated extra search costs (time) in finding these 'greener pastures' may cause that the quantity of food gathered is lower than the imitating agents gather.

After the consumption of opportunities, a new level of need satisfaction will be derived, and changes will occur regarding consumats' abilities, opportunities and uncertainty. Moreover, the environment the consumats behave in, e.g. a collective resource, will change as a consequence of their behaviour, thereby affecting the 
behaviour in subsequent time steps. The full consumat model is being depicted in the following Figure 4.

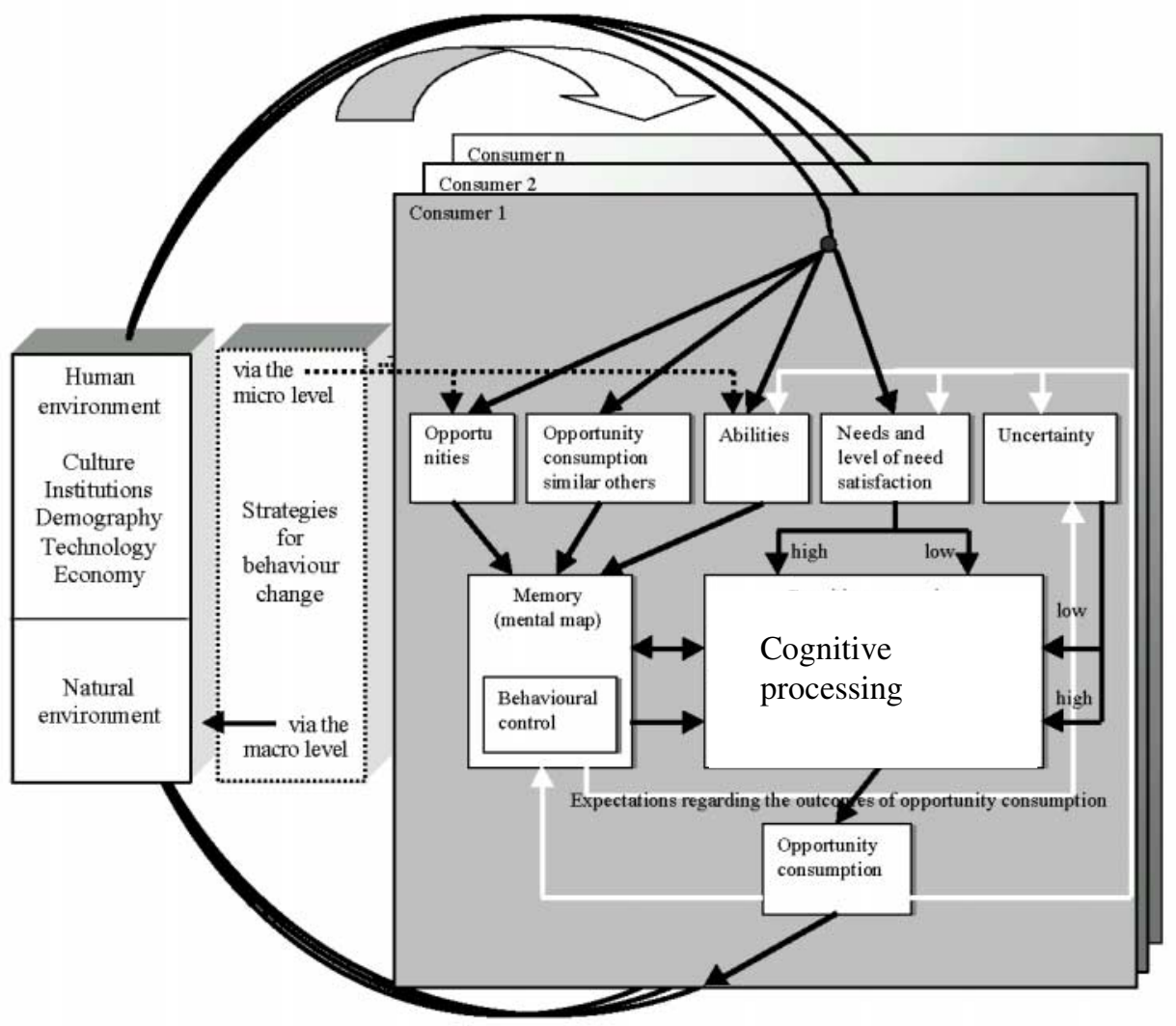

Fig. 4. The full consumat model

Working with the consumat approach on different topics has led us to some conclusions that may hold for the use of a basic agent structure in general. First of all, the results of experiments on different topics are often easy to relate. For example, when identified an 'imitation effect' in a series of relative simple simulation runs, it appeared that this effect also could explain part of the results we found in simulation runs where we formalise a more complex world. Hence the common basic structure facilitates the interchange of experimental results. Especially when the formalisation of the system varies with regard to complexity, it may be so that experiments with a simple system may reveal dynamics that contribute to interpreting the results obtained with a more elaborate formalisation of a system.

A second advantage we experienced working with our consumat approach is that it appeared to be easy to formalise adaptations to study the effects of certain variables. As such we have done experiments in which we formalised Social Value 
Orientation (e.g. individualistic, cooperative) in agents by adapting the formalisation of needs [36]. In experiments on preferences we explored the effects of assuming that socialisation and repetitive consumption would alter the preferences agent have for certain opportunities [34]. In [32] we experimented with learning, by assuming that consumats may discover more effective fishing techniques when they deliberate, which subsequently may spread through the population by social processes. Currently we are experimenting with consumats that generate offspring when successful, which allows for experimenting the evolution of consumat characteristics.

A third advantage is that data from laboratory experiments may be used in calibrating the rules of the agents. Whereas it appeared that it is hard to compare micro-level data of artificial agents with data from real subjects [36], it appeared that this comparison stimulated our thinking about which needs and processes should be incorporated in the model. More importantly, a perspective emerged of combining empirical and simulation studies as to explore the dynamics of e.g. simple resource systems, and provide a validation of the agent rules which can not be obtained when modelling more complex systems.

All these experiences convinced us of the value of using an integrated agent model when modelling behaviour in more complex systems.

We also found a number of disadvantages. Our models like many MAS models are artificial worlds, and are limited to explain real world phenomena. But starting to use micro-level data, we are confronted with the problem that a number of our hypotheses cannot be empirically tested because the appropriate data are not available. To strive towards such a validation at the micro level would require rigorously studying the use of heuristics in laboratory and field settings.

\section{Prospects of Integrated Agent Models of Behaviour}

The simulation of behaviour in systems is increasingly being recognised as a valuable tool to explore the dynamics of various systems. It is very important to capture the relevant micro-level dynamics in these models. For example, if a MAS-model is being used for policy development and support, it may be dangerous to base suggestions for policy measurements on false assumptions of the micro-level behavioural dynamics. To increase the validity of the behavioural dynamics as generated by artificial agents it is recommended to use a basic structure of the agent structure that is based on empirical findings in social sciences such as economics, psychology, sociology, political science and anthropology. Using such a basic structure also facilitates the discussion on how to formalise agent rules in a certain context, and contributes to the comparability of simulation results. Up till now the discussion is often unclear because of the fundamental differences in the behavioural architecture of agents, and hence the incomparability of results. Using a joint basic structure, how different the eventual formalisations may be, will contribute to the exchange of information between research groups, and hence benefit both the growth of knowledge on human dynamics in complex systems, and the application of this knowledge.

Including behavioural dynamics in a more empirically grounded way in simulation models of more complex systems would also allow for experimenting with 
real people managing artificial systems. Hence it would be possible to experimentally study the management of e.g. large organisations or ecosystems including policy measures aimed at changing the behaviour of the simulated agents.

Acknowledgment. The second author gratefully acknowledges support from the Center for the Study of Institutions, Population, and Environmental Change at Indiana University through National Science Foundation grants SBR9521918 and SES0083511.

\section{References}

1. Reynolds, C.W. (1987) Flocks, Herds, and Schools: A Distributed Behavioral Model, Computer Graphics, 21(4): 25-34.

2. Camazine, S. J.-L. Deneubourg, N.R. Franks, J. Sneyd, G. Theraulaz and E. Bonabeau (2001). Self-Organization in Biological Systems, Princeton University Press.

3. Schelling, T.C. (1971), 'Dynamic Models of Segregation', Journal of Mathematical Sociology, 1: 143-186.

4. Axelrod, R. (1984), The evolution of cooperation, Basic Books, New York.

5. Erev, I., A. Roth (1998) Predicing How People Play Games: Reinforcement Learning In Experimental Geamas with Unique, Mixed Strategy Equilibria, American Economic Review 88: 848-881.

6. Duffy, J. (2001). Learning to speculate: Experiments with artificial and real agents. Journal of Economic Dynamics \& Control, 25: 295-319.

7. Hommes, C.H., (2001). Financial Markets as Nonlinear Adaptive Evolutionary Systems, Quantitative Finance, 1: 149-167.

8. Gigerenzer, G., P.M. Todd, and the ABC Research (1999), Simple Heuristics That Make Us Smart. New York: Oxford University Press.

9. Gintis, H. (2000), 'Beyond Homo economicus: evidence from experimental economics', Ecological Economics, 35: 311-322.

10. Selten, R. (2001) What Is Bounded Rationality?, in Gigerenzer, G, and R. Selten (eds). Bounded Rationality: The Adaptive Toolbox, Cambridge, MA: MIT Press, pp.13-36.

11. Khalil, E.L. (1996). Social theory and naturalism. An introduction. In: E.L.Khalil and K.E. Boulding (Eds.). Evolution, order and complexity. London: Routledge. pp. 1-39

12. Bousquet, F., Cambier, C., Mullon, C., Morand P. and Quensiere, J., (1994). Simulating fishermen's society. In: N. Gilbert and J. Doran (Eds.) (1994). Simulating societies: The computer simulation of social phenomena. London: UCL Press.

13. Bousquet, F., O. Barretau, P. d'Aquino M. Etienne, S. Boissau, S. Aubert, C. Le Page, D. Babin and J.C. Castella (2002) Multi-agent systems and role games : collective learning processes for ecosystem management, , in Janssen, M.A. (ed.) Complexity and Ecosystem Management: The Theory and Practice of Multi-agent Systems, Edward Elgar Publishers, Cheltenham UK/ Northampton, MA, USA.

14. Mosler, H.J., Schwarz, K., Ammann, F., Gutscher, H. (2001) Computer simulation as a method of further developing a theory: Simulating the Elaboration Likelihood Model. Personality and Social Psychology Review, 5 (3): 201-215.

15. Wilk, R. (2002) Consumption, human needs, and global environmental change, Global Environmental Change, 12: 5-13.

16. Vallacher, R.R. and Nowak, A., (1994). The chaos in social psychology. In: R.R. Vallacher and A. Nowak, (Eds.). Dynamical systems in social psychology. San Diego: Academic Press, Inc.

17. Maslow, A.H. (1954). Motivation and Personality. New York, USA: Harper and Row. 
18. Max-Neef, M., (1992). Development and human needs. In: P. Ekins and M. Max-Neef (Eds.): Real-life economics: Understanding wealth creation. London, New York: Routledge.

19. Sauermann, H., \& R. Selten (1962). Anspruchsanpassungstheorie der Unternehmung. Zeitschrift für die gesamte Staatswissenschaft. 118: 577-597.

20. Selten, R. (1998). Aspiration Adaptation Theory. Journal of Mathematical Psychology. 42: 191-214.

21. Buss, D.M. (1997) Evolutionary Foundations of Personality. In: R. Hogan, J. Johnson, and S. Briggs (Eds.), Handbook of personality psychology (pp. 317-344). London: Academic Press.

22. Simon, H.A. (1976). Administrative behavior: a study of decision-making processes in administrative organizations. New York: Harper.

23. Jager, W., (2000). Modelling consumer behaviour. Doctoral thesis. Groningen: University of Groningen, Centre for Environmental and Traffic psychology. http://docserver.ub.rug.nl/eldoc/dis/ppsw/w.jager/

24. Jager, W., \& Janssen, M.A. (2002). How to decide how to decide: An integrated and dynamical perspective on human decision-making. Working paper: University of Groningen, Faculty of Management and Organisation, Dept. of Marketing.

25. Payne, J.W., Bettman, J.R., \& Johnson, E.J. (1993). The adaptive decision maker. New York: Cambridge University Press.

26. Weiss, G., \& Sen, S. (Eds.)(1996). Adaptation and learning in multi-agent systems. Lecture Notes in Artificial Intelligence, Volume 1042. Heidelberg: Springer-Verlag

27. Weiss, G. (Ed.) (1997). Distributed artificial intelligence meets machine learning: Learning in multi-agent environments. Lecture Notes in Artificial Intelligence, Volume 1221. Heidelberg: Springer-Verlag

28. Conte, R., (1999). Social Intelligence Among Autonomous Agents. Computational and Mathematical Organization Theory 5(3), 203-228.

29. Conte, R. \& Paolucci, M. (2001). Intelligent Social Learning. Journal of Artificial Societies and Social Simulation vol. 4, no. 1, http://www.soc.surrey.ac.uk/JASSS/4/1/3.html

30. Hebb, D.O. (1949). The Organization of Behavior: New York: John Wiley and Sons, Inc.

31. Jager, W., Janssen, M.A. and C.A.J. Vlek (1999). Consumats in a commons dilemma: Testing the behavioural rules of simulated consumers. COV report no. 99-01. 56 pages. Groningen: Centre for Environment and Traffic Psychology, University of Groningen

32. Jager, W., Janssen, M.A, De Vries, H.J.M., De Greef, J. and Vlek, C.A.J. (2000). Behaviour in commons dilemmas: Homo Economicus and Homo Psychologicus in an ecological-economic model. Ecological Economics, Vol. 35:357-380.

33. Jager, W., Janssen, M., \& Vlek, C., (in press, 2002). How uncertainty stimulates overharvesting in a commons dilemma: the imitation effect. Journal of Environmental Psychology.

34. Janssen, M.A. and Jager, W., (2001). Adoption of new products in a market of heterogeneous and changing preferences and social networks Journal of Economic Psychology, 22: 745-772.

35. Janssen, M.A. \& Jager, W. (in press 2002) Stimulating diffusion of green products. Coevolution between firms and consumers. Journal of Evolutionary Economics

36. Jager, W., Janssen, M.A. (in press 2002). Using artificial agents to understand laboratory experiments of common-pool resources with real agents, in Janssen, M.A. (ed.) Complexity and Ecosystem Management: The Theory and Practice of Multi-agent Systems, Edward Elgar Publishers, Cheltenham UK/ Northampton, MA, USA

37. Festinger, L. (1954). A theory of social comparison processes. Human Relations, 7, 117-140.

38. Hofstee, W. K. B., De Raad, B., \& Goldberg, L. R. (1992). Integration of the Big Five and Circumplex approaches to trait structure. Journal of Social and Personality Psychology, $63,146-163$. 